\title{
AUTOMATED SPECTRAL CLASSIFICATION OF STARS BY MEANS OF OBJECTIVE PRISM SPECTRA
}

\author{
T.SHVELIDZE ${ }^{1}$ AND V.MALYUTO \\ ${ }^{1}$ Abastumani Astrophysical Observatory, Georgia; \\ ${ }^{2}$ Tartu Astrophysical Observatory, Estonia
}

Some years ago a complex program of studying the main meridional section of the Galaxy was initiated with the aim of improving our knowledge of spatial and kinematic characteristics of stellar populations. To classify stars, objective prism stellar spectra ( $\mathrm{D}=166 \mathrm{~A} / \mathrm{mm}$ at $\left.H_{\gamma}\right)$, are used. The field diameter is $4^{\circ} 50^{\prime}$, the limiting photographic stellar magnitude is about $12^{m}$. Our automated quantitative spectral classification of F-K stars applies criteria evaluation and is based mainly on the SDR package for spectrophotometric data reduction (Malyuto, Pelt, Shvelidze, 1993) and the CTATEC-2 package for the definition of a multiple linear regression model "criteria values versus main physical parameters" (Malyuto, Shvelidze, 1989). Our regression model was based on the final sample of calibration stars containing 95 standard (bright) stars and 96 program faint $\left(8^{m}<B<11^{m} .6\right)$ stars from our areas near the North Galactic Pole. The standard deviations of our calibration with the use of all data taken together are \pm 0.015 for $\log \mathrm{T}_{\text {eff }}, \pm 0^{m} .96$ for $\mathrm{Mv}$ and \pm 0.25 for $[\mathrm{Fe} / \mathrm{H}]$. These results are encouraging for application of our method to a large set of Abastumani objective prism spectra.

\section{References}

Malyuto , V., Shvelidze, T. (1989) The technique of automatic quantitative stellar spectral classification using stepwise linear regression, Astrophysics and Space Sci., 155, pp. 71-83

Malyuto, V., Pelt, J., Shvelidze, T. (1993) The spectrophotometric data reduction software package for automated quantitative spectral classification of stars, Baltic Astronomy, 1, pp. 526-544 